\title{
Prostanoids with Cyclopentenone Structure as Tools for the Characterization of Electrophilic Lipid-Protein Interactomes
}

\author{
KONSTANTINOS STAMATAKIS AND DOLORES PÉREZ-SALA \\ Departamento de Estructura y Función de Proteínas, Centro de Investigaciones \\ Biológicas, C.S.I.C., 28040 Madrid, Spain
}

\begin{abstract}
Electrophilic eicosanoids arise from the free radical-induced peroxidation of arachidonic acid or its metabolites. These reactive species may play an important role in pathophysiological processes associated with inflammation and oxidative stress. Cyclopentenone prostaglandins (cyPG) and isoprostanes are reactive eicosanoids that can form covalent adducts with cysteine residues in proteins through Michael addition. In pharmacological studies, cyPG have shown potent protective effects in experimental models of inflammation and tissue injury, and they have been proposed to contribute to inflammatory resolution. An important mechanism for the anti-inflammatory effects of cyPG is the covalent modification of critical cysteine residues in proteins involved in the modulation of inflammation, such as transcription factors NF- $\mathrm{B}$ and AP-1. In recent years, analogs of electrophilic prostanoids have been used in various approaches to identify biologically relevant protein targets for this modification. Prostanoids with cyclopentenone structure have been shown to target a defined subproteome that is beginning to be characterized. Structural studies suggest that diverse cyPG may modify distinct proteins selectively. Functional studies put forward a dual role for these compounds in the cellular response to inflammation or stress. Therefore, a detailed knowledge of targets of electrophilic eicosanoids and the functional consequences of their modification will contribute to the understanding of their mechanism of action and help assess whether these endogenous mediators can be exploited as the basis for the development of novel therapeutic strategies. In this article we discuss the recent advances in this rapidly growing field.
\end{abstract}

KEYWORDS: cyclopentenone prostaglandins; proteomics; posttranslational modification; inflammation; cysteine

Address for correspondence: Dr. Dolores Pérez-Sala, Centro de Investigaciones Biológicas (C.S.I.C.), Ramiro de Maeztu, 9, 28040 Madrid, Spain. Voice: 34-91-8373112; ext.: 4212; fax: 3491-5360432.

e-mail:dperezsala@cib.csic.es

Ann. N.Y. Acad. Sci. 1091: 548-570 (2006). (C) 2006 New York Academy of Sciences. doi: 10.1196/annals.1378.096 


\section{INTRODUCTION}

The formation of reactive species in living cells is a process cosubstantial with normal cell activity and it can be increased under pathophysiological conditions. These reactive intermediates are required for normal cell metabolism and gene expression and constitute important players in cell signaling. However, they can also damage cellular components, like DNA and proteins. ${ }^{1}$ Reactive intermediates can be derived from the use of oxygen by living cells, like superoxide anion or hydrogen peroxide, nitric oxide (NO), and peroxynitrite. These species can act through a wide array of mechanisms including their reaction with other cellular components, such as amino acids, lipids, or sugars, which gives rise to various electrophiles with diverse biological activities. The electrophiles generated can interact with chemical groups of nucleophilic character, such as the thiol groups present in cysteine residues of proteins or in the tripeptide glutathione (GSH). The modification of cysteine residues by these diverse species completely alters their physicochemical properties and has important functional consequences. For these reasons much attention has been devoted in recent years to the identification and characterization of the structural and functional consequences of the modification of cysteine residues by diverse reactive species under physiological or pathophysiological conditions. The group of proteins involved in the regulation of cellular functions through the modification of their cysteine residues could be termed sulfoproteome. Methods for the detection of specific cysteine modifications by thiolation or nitrosylation have been developed. ${ }^{2,3}$ These methods have allowed the identification of proteins which are targets for these modifications and in some cases, the identification of novel signaling pathways. In addition to its role in biological pathways, the modification of cysteine residues has been considered of great importance for the design of novel antiviral and anticancer therapies. ${ }^{4}$ During the past 5 years several reports have highlighted the importance of the modification of cysteine residues by electrophilic eicosanoids. This type of modification has remarkable pharmacological consequences and its potential physiological relevance is beginning to be unveiled. The available evidence indicates that the direct modification of cysteine residues contributes to the effects of electrophilic eicosanoids on the onset and the resolution of inflammation, and it could provide connections between inflammatory situations and increased proliferation. An understanding of these processes requires the identification and characterization of the electrophilic eicosanoid interactome.

\section{EICOSANOIDS AND THEIR IMPORTANCE IN INFLAMMATION}

Eicosanoids are lipidic mediators derived from the essential fatty acid arachidonic acid that are involved in the regulation of multiple cellular processes, 
ranging from the regulation of vascular tone, to the modulation of gene expression and cell proliferation. Arachidonic acid is mainly found esterified to membrane phospholipids. A variety of stimuli, including inflammatory agents or tissue injury, activate phospholipases, mainly phospholipase $\mathrm{A}_{2}$, that release arachidonic acid from membranes. Arachidonic acid can then be transformed through enzymatic and nonenzymatic reactions into a wide variety of biologically active compounds. Cyclooxygenase (COX) enzymes, catalyze the limiting step in the synthesis of prostaglandins (PG), that is, the formation of $\mathrm{PGH}_{2}$. This compound is then transformed by various $\mathrm{PG}$ synthases into other PG with specific functions, and thromboxane $A_{2}$ (reviewed in Ref. 5). Arachidonic acid can be transformed into leukotrienes and lipoxins through the action of lipoxygenase (LOX) enzymes. ${ }^{6}$ In addition, arachidonic acid and its derivatives can suffer various nonenzymatic transformations, like free radical-induced peroxidation, which gives rise to a series of compounds that are known as isoprostanes. ${ }^{7}$ It is interesting to note that significant amounts of PG can also be generated through nonenzymatic mechanisms. ${ }^{8} \mathrm{PG}$, in turn, can suffer nonenzymatic dehydration to yield PG with cyclopentenone structure (cyPG). ${ }^{9}$

Inflammation is a complex response characterized by multiple interactions between soluble and cellular factors that tend to defend the organism against traumatic, infectious, or autoimmune injury. At the site of tissue injury, a complex array of substances can be released, including neuropeptides, intracellular materials, or microbial antigens, which activate resident cells, such as mast cells and macrophages. These cells in turn release inflammatory mediators (histamine, cytokines, NO, PG, etc.) that induce vasodilatation and edema and contribute to attract neutrophils, which produce large amounts of proteases and reactive oxygen species, and lymphocytes that contribute to activate macrophages. During inflammation there are marked alterations in gene expression. Proinflammatory stimuli induce the expression and activation of proteins involved in the synthesis of inflammatory mediators. Inducible NO synthase and COX-2 play a key role in the increased synthesis of $\mathrm{NO}$ and PG during inflammation. Both types of mediators can amplify the response to proinflammatory stimuli at the early stages of the inflammatory response. ${ }^{10,11}$ Thus, there are various self-amplifying mechanisms, which operate during the onset of the inflammatory response and promote a controlled destruction of tissue in order to remove the damaging agent. However, for this situation to lead to healing, the mechanisms for tissue repair must be in place. The mechanisms of resolution of inflammation are active and tightly regulated processes that favor the safe disposal of inflammatory cells and the restoration of the inflamed tissue to its physiological situation. ${ }^{12}$ During the resolution phase of inflammation an important event takes place, referred to as "class switching," that affects the nature of the inflammatory mediators. This implies a reduction in proinflammatory and an increase in anti-inflammatory mediators that contribute to limit the amplitude of the response. In addition, several well-known 
inflammatory mediators can display dual effects, that is, they exert mainly proinflammatory actions during the early phases of the inflammatory response and inhibitory actions at late stages. An example of this is NO, for which we described recently a biphasic effect on the response of mesangial cells to cytokine stimulation. ${ }^{13} \mathrm{~A}$ dual nature has also been reported for other mediators like tumor necrosis factor- $\alpha$ (TNF- $\alpha$ ), interferon- $\gamma$ (IFN- $\gamma$ ), transforming growth factor- $\beta$ (TGF- $\beta$ ), and some eicosanoids, such as PGE $_{2} .{ }^{14}$

Eicosanoids play a key role both in the onset and in the resolution of inflammation. PG and leukotrienes display potent proinflammatory effects, including vasodilatation, edema, fever, activation of neutrophil chemotaxis, increased expression of adhesion molecules, etc. ${ }^{5}$ Isoprostanes and some of their derivatives are considered an index of oxidative stress and lipid peroxidation. ${ }^{15}$ Some isoprostane-derived products can contribute to oxidative damage through the modification of amino acid residues in proteins that may result in protein aggregation. ${ }^{16}$ In contrast, cyPG and lipoxins appear to display mostly antiinflammatory effects, including the inhibition of proinflammatory cytokine synthesis and neutrophil chemotaxis and transmigration, and the induction of neutrophil clearance through phagocytosis by monocytes-macrophages. ${ }^{17}$ Eicosanoid production plays an important role in the process of "class switching." It has been reported that cyPG are generated in increased amounts at the late stages of inflammation. ${ }^{18}$ In addition, lipoxins are generated through the transcellular metabolism of arachidonic acid, that is, arachidonic acid or its products generated by a class of cells are taken up by a different class, which can convert them in lipoxins through the action of 12- or 15-LOX. ${ }^{12,17}$ This represents a mechanism by which cell-cell interactions favor a transition in the profile of the eicosanoids produced, from a proinflammatory to an antiinflammatory nature, and this has important implications for the resolution of inflammation.

\section{CYCLOPENTENONE PROSTAGLANDINS}

cyPG are electrophilic eicosanoids that have elicited great interest recently. Their common feature is the presence of an unsaturated carbonyl group in the cyclopentane ring (cyclopentenone, see FIG. 1). CyPG are generated by spontaneous dehydration of other PG. Thus, $\mathrm{PGA}_{2}$ arises by dehydration of $\mathrm{PGE}_{2}$, while cyPG of the $\mathrm{J}$ series, including $\mathrm{PGJ}_{2}$ and 15deoxy- $\Delta^{12,14}-\mathrm{PGJ}_{2}\left(15 \mathrm{~d}-\mathrm{PGJ}_{2}\right)$ arise from $\mathrm{PGD}_{2}$. It has been known for over 20 years that cyPG exert antiviral and antiproliferative effects. However, a renewed interest in this area rose when $15 \mathrm{~d}-\mathrm{PGJ}_{2}$ was identified as an endogenous ligand of the transcription factors of the PPAR (peroxisome proliferator activated receptor family), ${ }^{19}$ which play an important role in the regulation of lipid metabolism and immune response. In fact, activation of PPAR has been reported to mediate some of the beneficial effects of 
(A)

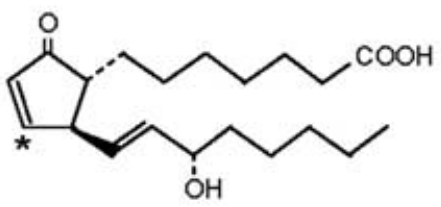

$\mathrm{PGA}_{1}$<smiles>CCCCCC(O)C=C[C@H]1C=CC(=O)[C@@H]1CC=CCCCC(=O)O</smiles>

$\mathrm{PGA}_{2}$<smiles>CCCCCC(O)/C=C/[C@H]1C(=O)C=C[C@@H]1C/C=C/CCCC(=O)O</smiles>

$P \mathrm{PJ}_{2}$

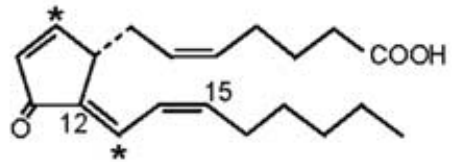

15-deoxy- $\Delta^{12,14}-\mathrm{PGJ}_{2}$

(B)

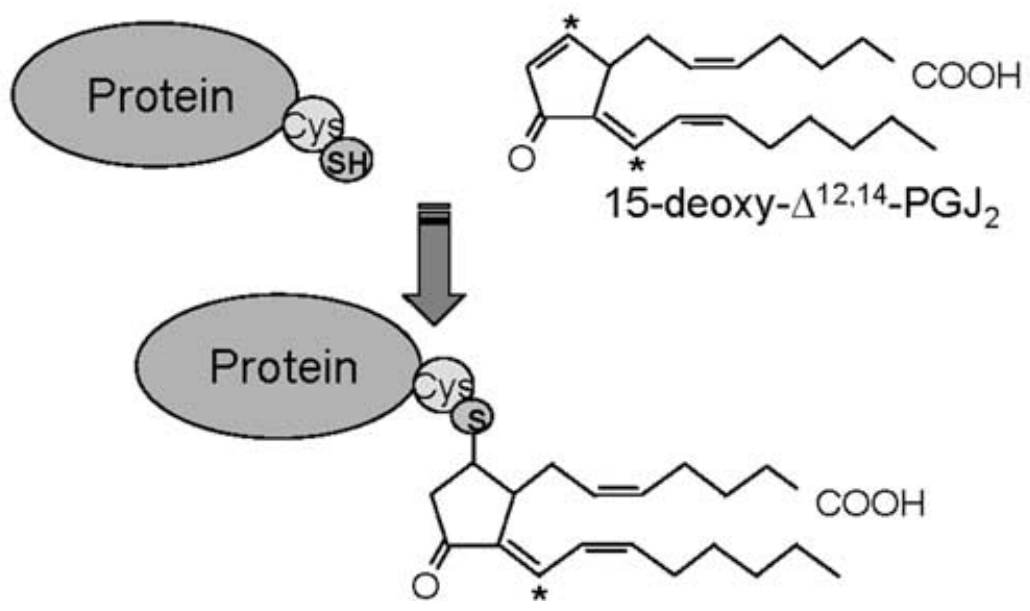

FIGURE 1. Structure of cyPG and formation of Michael adducts with proteins. (A) Structures of several cyPG of the A series (left) and of the J series (right). Electrophilic carbons are marked by asterisks. (B) Schematic representation of the formation of a Michael adduct between the electrophilic carbon in the cyclopentenone ring of $15 \mathrm{~d}_{-} \mathrm{PGJ}_{2}$ (carbon 9) and the sulphydryl group of a protein cysteine residue.

$15 \mathrm{~d}-\mathrm{PGJ}_{2}$ in experimental models of inflammation. However, it has been found that many of the anti-inflammatory effects of cyPG can occur by mechanisms independent from the activation of PPAR. ${ }^{9}$ These effects include the inhibition of transcription factor NF- $\mathrm{KB}$ and of the induction of several proinflammatory genes, including iNOS, COX-2, or ICAM-1. ${ }^{20,21}$ Some of the effects of cyPG could be observed in cell-free systems ${ }^{22}$ and required the presence of critical 
cysteine residues in NF- $\mathrm{KB}$ or in IKK. ${ }^{23,24}$ For these reasons it was postulated that these inhibitory effects could be mediated by the direct interaction of cyPG with cysteine residues by Michael addition. In this context, our group provided the first direct evidence of the interaction between cyPG and a protein target, the p50 subunit of transcription factor NF- $\mathrm{B}$, which we demonstrated by the use of a biotinylated analog of $15 \mathrm{~d}-\mathrm{PGJ}_{2}$ combined with mass spectrometry analysis. ${ }^{24}$ Several research groups have employed similar strategies to identify other targets of cyPG or of compounds with similar reactivity. Some of the identified targets play important roles in the modulation of inflammatory and proliferative processes. Anti-inflammatory cyPG may interact with the NF- $\mathrm{B}$ activation pathway at multiple levels (FIG. 2), including the inhibition of the activity of the proteasome, ${ }^{25}$ the modification of critical cysteines in IKK $\alpha$ and $\beta,{ }^{22,23}$ and also modification of cysteines present in the DNA binding

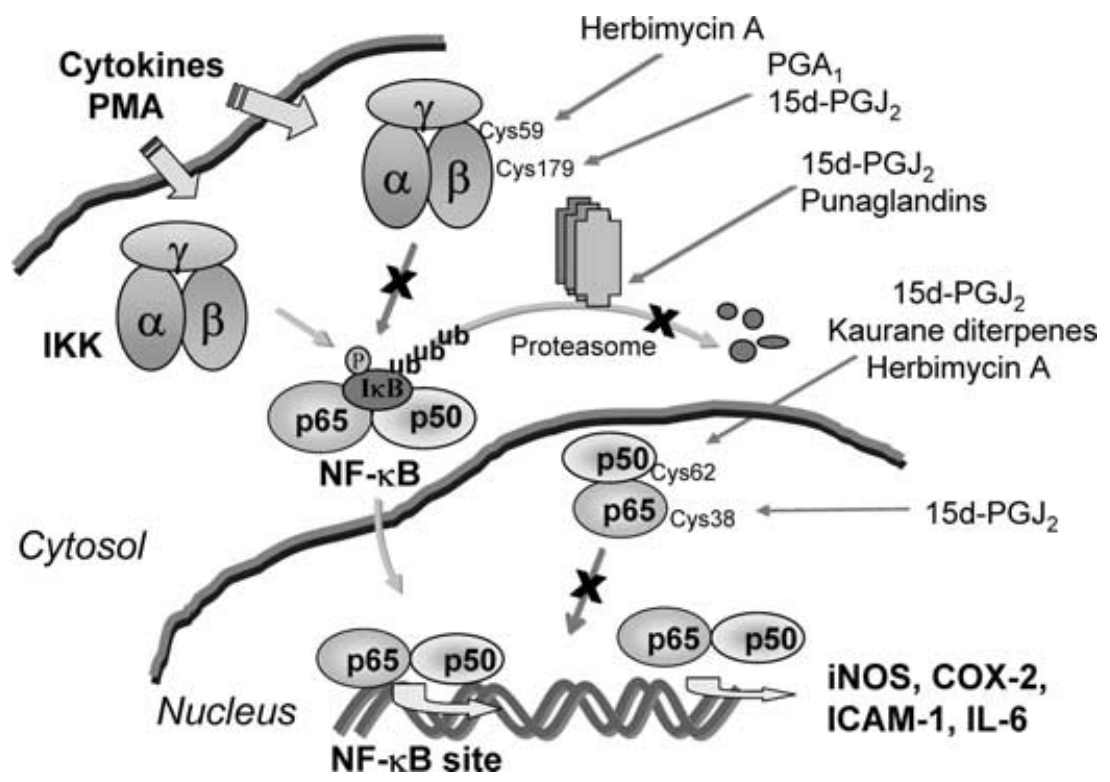

FIGURE 2. Schematic view of the NF-кB activation pathway showing the sites of interaction of several Michael receptors. The transcription factor NF- $\kappa$ B plays a key role in the inflammatory response. In a very schematic view, the pathway leading to NF- $\mathrm{B}$ activation involves the stimulation of a complex with kinase activity (IKK), which phosphorylates the

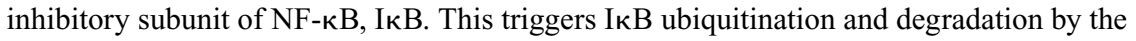
proteasome, which promotes the nuclear accumulation of the active dimer, represented by p65/p50, which activates the transcription of target genes. The NF- $\kappa$ B activation pathway has been the subject of numerous studies in search for anti-inflammatory molecules. Shown is the site of action of several molecules that have been reported to interfere with NF- $\kappa \mathrm{B}$ activation through the formation of covalent adducts with critical proteins of this pathway by Michael addition. 
domains of the subunits of this transcription factor (p65 and p50). ${ }^{24,26}$ As a result of these interactions, cyPG can inhibit the phosphorylation and/or the degradation of the inhibitory subunit IкB, and block the translocation or the nuclear accumulation of the active factor. In addition, they may directly inhibit the binding of NF- $\mathrm{BB}$ proteins to DNA. The predominant effect would be dependent on the cell type. $15 \mathrm{~d}-\mathrm{PGJ}_{2}$ can also target the cysteine residue present in the DNA binding domain of c-Jun, and thus, inhibit the DNA binding activity of AP- $1 .{ }^{27}$ In addition, some cyPG can bind to and inactivate thioredoxin and thioredoxin reductase, ${ }^{28,29}$ which are involved in the control of cellular redox status and play a role in maintaining the cysteines of NF- $\mathrm{KB}$ and AP-1 in their reduced state, which is required for function. Therefore, the modification of these redox-controlling proteins could also have consequences for the modulation of the inflammatory response. Several electrophilic lipids, including $15 \mathrm{~d}-\mathrm{PGJ}_{2}$ can also activate the transcription factor Nrf2, through the modification and inhibition of the function of Keap1, a cytoplasmic protein that acts as an inhibitor of Nrf2. Through this mechanism cyPG can induce the expression of hemooxygenase and of antioxidant genes..$^{30,31}$ Other identified targets of cyPG include H-Ras and LKB1/STK11 (serine/threonine kinase 11), proteins involved in the control of cellular proliferation. ${ }^{32,33}$ For these reasons, cyPG have been proposed to participate in the interactions linking chronic inflammation and increased cell proliferation.

\section{cyPG: Are they Physiologically or Pharmacologically Relevant?}

The characterization of the biological effects of cyPG has evolved in parallel with the exploration of their potential therapeutic use in various experimental models of inflammation and tissue injury. $15 \mathrm{~d}-\mathrm{PGJ}_{2}$ has been reported to display protective effects against renal ischemia-reperfusion injury, ${ }^{34}$ adjuvantinduced arthritis, ${ }^{35}$ experimental autoimmune encephalomyelitis, ${ }^{36}$ restenosis after angioplasty, ${ }^{37}$ etc. In some of these models, the beneficial effects have been found to correlate with the inhibition of the activity of transcription factors NF- $\mathrm{kB}$ and AP-1, and with the ability of cyPG to covalently modify proteins. Interestingly, animals deficient in $\mathrm{PGD}_{2}$ synthase show exacerbated inflammation, thus pointing toward a protective role of $\mathrm{PGD}_{2}$ metabolites in inflammation. ${ }^{38}$ However, the physiological relevance of the effects of cyPG is not established. This is due to the fact that the concentrations of cyPG required to elicit anti-inflammatory effects are generally higher than those measured in vivo. This discrepancy could be due to the high reactivity of cyPG that readily form adducts with thiol groups, making the detection of their total levels in biological samples inaccurate. It also should be taken into account that the protective effects of cyPG are not general. In some tissues, cyPG can have toxic effects, ${ }^{39}$ or induce unwanted reactions, such as allergy or cell 
proliferation. For these reasons, the potential therapeutic use of cyPG requires the identification of their targets and the assessment of their selectivity.

\section{CYCLOPENTENONE ISOPROSTANES}

In contrast to cyPG, the available information regarding the generation of isoprostanes in biological systems is more comprehensive. Roberts and Morrow first reported that free radical-induced peroxidation of arachidonic acid could lead to the nonenzymatic formation of bioactive $\mathrm{PG} \mathrm{F}_{2}$-like compounds, $\mathrm{F}_{2}$-isoprostanes, in vivo. Later, several compounds were identified that could be formed by the isoprostane pathway (reviewed in Ref. 15). It is known that isoprostane levels are greatly increased in various tissues under conditions of oxidative stress or chronic inflammation, including Alzheimer, atherosclerosis, and rheumatoid arthritis. In a way analogous to that described for cyPG, nonenzymatic dehydration of isoprostanes generates compounds with cyclopentenone structure, which are highly reactive and have been found in tissues at near micromolar concentrations. ${ }^{40} \mathrm{~A}_{2}$ and $\mathrm{J}_{2}$ isoprostanes are isomers of the corresponding cyPG. In a recent work, ${ }^{41} \mathrm{~A}$ and $\mathrm{J}$ series isoprostanes have been found to mimic the effects of cyPG on the inflammatory response in macrophages. These compounds can inhibit NF-кB, induce apoptosis of macrophages, and in the case of the J series, activate PPAR $\gamma$. These observations suggest that cyclopentenone isoprostanes could act as negative modulators of inflammation. In a way analogous to $15 \mathrm{~d}-\mathrm{PGJ}_{2}$, the isoprostane $15-\mathrm{A}_{2}$-isoprostane has been reported to modify Keap1 and activate Nrf2. ${ }^{30}$ The availability of isoprostanes through chemical synthesis will grant a spur in the research into their biological effects.

\section{LIPIDIC MEDIATORS AND OTHER COMPOUNDS WITH UNSATURATED CARBONYL GROUPS}

There are other lipidic mediators, that can arise under situations of inflammation or oxidative stress, which possess unsaturated carbonyl groups and may form covalent adducts with thiol groups. Among these compounds are 5- and 15-eicosatetraenoid acid, 9- and 13-oxo-octadienoic acid, ${ }^{42}$ several 15-keto-PG, epoxycyclopentenone phospholipids, ${ }^{43,44}$ and neuroprostanes. ${ }^{39}$ These lipids act through various mechanisms, in some cases not completely understood. Also, the well-studied $\alpha, \beta$-unsaturated aldehydes 4-hydroxy-nonenal and acrolein can be formed as products of lipid peroxidation and can react both with thiol groups by Michael addition and with amine groups forming Schiff bases. Several excellent reviews ${ }^{45,46}$ have dealt with the biological effects of these reactive aldehydes; therefore, this subject will not be considered here in more detail. In several studies, 4-hydroxy-nonenal has been shown to modify protein targets common to cyPG. ${ }^{29,30,33}$ The predominance of protective or 
toxic effects of these electrophilic compounds appears to be concentrationdependent and cell-type specific. It has been proposed that moderate levels of oxidized lipids elicit an adaptative response to stress that protects cells from subsequent oxidative insults, while high concentrations of electrophilic lipids would induce apoptosis. ${ }^{47}$ In addition to endogenous compounds, many natural products, like active principles from medicinal herbs, possess unsaturated carbonyl groups in their structure, which may show reactivity similar to that of cyPG. For instance, parthenolide or the kaurane diterpene kamebakaurin, ${ }^{48,49}$ inhibit NF- $\mathrm{B}$ activation through mechanisms analogous to those of cyPG (FIG. 2). In addition, herbimycin $\mathrm{A}$ and punaglandins have been reported to inhibit NF- $\kappa$ B through the formation of Michael adducts with critical cysteine residues of components of the NF- $\mathrm{kB}$ activation pathway. ${ }^{50-52}$ For these reasons, certain natural or synthetic cyclopentenones are being studied in order to assess their potential use as antiproliferative or anti-inflammatory agents.

\section{DERIVATIVES OF CYPG AS TOOLS FOR THE STUDY OF THE INTERACTIONS BETWEEN ELECTROPHILIC EICOSANOIDS AND CELLULAR PROTEINS}

Eicosanoids with cyclopentenone structure have been used in various approaches to understand the mechanism of action of electrophilic lipids. The synthesis of cyPG allowed to explore the interactions of these compounds with cellular structures and with proteins. ${ }^{53}$ The interaction of cyPG with cells was explored in a series of important studies by Narumiya and co-workers. ${ }^{54-56}$ Using radioactively labeled cyPG, these authors showed that these compounds could enter intact cells in significant amounts and that they could be found both in cytosolic and nuclear compartments, with preferential accumulation in cell nuclei, where cyPG were bound to an insoluble fraction. They found that the retained radioactivity could be recovered by treatment with alkali and by proteolytic digestion of the nuclear fraction, which suggested the formation of Michael adducts between cyPG and nuclear proteins. ${ }^{56}$ In addition, these authors showed that the extent of the accumulation was dependent on the structure of the cyPG. In the case of $\mathrm{PGA}_{2}$ both the binding to cellular structures and the inhibitory effect of cell growth could be reversed by extensive washing. In contrast, nuclear accumulation of $\Delta^{12}-\mathrm{PGJ}_{2}$ was only partially reversed by cell washing and the growth inhibitory effect was irreversible. ${ }^{56}$ Therefore, this constitutes one of the first evidences of a differential effect of cyPG with diverse structure.

The use of a biotinylated analog of $\mathrm{PGA}_{2}$ provided one of the first evidences of the binding of cyPG to cellular polypeptides by means of SDSPAGE and Western blot with streptavidin-alkaline phosphatase. This allowed the visualization of several biotin-positive polypeptides whose labeling correlated with the inhibition of cellular proliferation exerted by $\mathrm{PGA}_{2}$ in $\mathrm{K} 562$ erythroleukemia cells. ${ }^{57} \mathrm{We}$ used a modification of this procedure to provide 
the first identification and visualization of a protein that could be modified through the stable binding of a biotinylated analog of $15 \mathrm{~d}-\mathrm{PGJ}_{2} \cdot{ }^{24}$ Since then, biotinylated analogs of cyPG have been used in several approaches to get insight into the identity and consequences of protein modification by cyPG. Biotinylated analogs of cyPG enter cells and form adducts with cellular proteins that are resistant to electrophoresis under denaturing conditions. Recently, $15 \mathrm{~d}-\mathrm{PGJ}_{2}$ modified by incorporation of a fluorescent moiety has been used to visualize the cellular sites of binding of this cyPG. ${ }^{58}$ It should be noted, however, that, although modified cyPG possess the reactive cyclopentenone moiety, their properties may not be identical to those of endogenous cyPG due to the presence of the incorporated label. For instance, in a recent work, several lipids containing $\alpha, \beta$-unsaturated ketone moieties have been reported to bind to PPAR $\gamma$ covalently. In this model, $15 \mathrm{~d}_{-} \mathrm{PGJ}_{2}$ has been proposed to occupy the ligand binding site in such a way that the carboxyl group of the PG establishes interactions with the PPAR backbone (formation of a hydrogen bond with Tyr-473 in helix 12 of PPAR $\gamma$ ), that are important for binding. ${ }^{42}$ This may provide an explanation for the observation that $15 \mathrm{~d}-\mathrm{PGJ}_{2}$ biotinylated at the carboxyl group did not detectably bind to PPAR $\gamma$ in cells, as explored with a pull-down assay, whereas it still bound to c-Jun or c-Fos. ${ }^{27}$ Taken together these evidences suggest that the structural requirements for covalent binding of $15 \mathrm{~d}-\mathrm{PGJ}_{2}$ to the PPAR $\gamma$ ligand binding domain are different from those involved in PPAR $\gamma$-independent actions. We recently described that the presence of the endocyclic double bond in $15 \mathrm{~d}-\mathrm{PGJ}_{2}$ was required for the efficient binding of this cyPG to c-Jun and to various proteins present in a mesangial cell lysate, in an in vitro assay, ${ }^{21}$ since an analog of $15 \mathrm{~d}-\mathrm{PGJ}_{2}$ lacking this double bond, namely 9,10-dihydro- $15 \mathrm{~d}_{-} \mathrm{PGJ}_{2}$ failed to mimic the effects of $15 \mathrm{~d}_{-}-\mathrm{PJ}_{2}$. This indicated that probably, carbon 9 of $15 \mathrm{~d}-\mathrm{PGJ}_{2}$ was involved in Michael addition with the thiol groups of the proteins studied. However, both $15 \mathrm{~d}-\mathrm{PGJ}_{2}$ and 9,10-dihydro-15d-PGJ ${ }_{2}$ bind to and activate PPAR $\gamma$ with similar potency, ${ }^{21}$ which indicates that the carbon important for the reaction with the sulfur atom of the cysteine residue in PPAR $\gamma$ is the carbon at position 13 , as suggested also by molecular modeling. ${ }^{42}$

\section{Use of Biotinylated Derivatives of Eicosanoids to Confirm the Interaction of cyPG with Potential Targets}

The observations described above indicate that electrophilic eicosanoids can potentially bind to additional or different protein targets than their modified counterparts. Therefore, care should be exercised when using these compounds because it would be important to confirm that natural and modified eicosanoids display the same mechanism of action for a particular target. In recent years, a number of reports have used biotinylated analogs of cyPG to assess the binding of these compounds to defined proteins in intact cells. This approach has been used to confirm the binding of cyclopentenone moiety containing 
compounds to the p50 subunit of NF- $\mathrm{kB},{ }^{24}$ the components of AP-1, c-Jun, and c-Fos, ${ }^{27}$ the protein thioredoxin, ${ }^{28}$ thioredoxin reductase, ${ }^{29} \mathrm{Keap} 1,{ }^{30}$ or $\mathrm{H}-$ Ras, ${ }^{32}$ among others. In some cases, it has been confirmed that the biotinylated cyPG mimics the biological effects of the nonmodified compound. ${ }^{24,30,59} \mathrm{In}$ addition, some studies infer the modification of the target cysteine residues by the natural electrophilic lipids in vitro or in intact cells, by evidencing a reduction in the ability of general cysteine reagents, such as biotinylated iodoacetamide or fluorescent maleimide, to modify the electrophile-treated proteins. ${ }^{21,30,42}$ However, it should be taken into account that treatment of cells with cyPG may induce potent oxidative stress, ${ }^{60-62}$ and this may be the cause for the occurrence of other modifications of cysteine residues, either reversible, such as thiolation, ${ }^{63}$ or irreversible, like the formation of sulfoxides, that could result in decreased accessibility of the cysteine residues for modification by thiol reagents. In addition, we have observed that treatment of human leukemia cells with $15 \mathrm{~d}_{-} \mathrm{PGJ}_{2}$ induces the formation of intermolecular and intramolecular disulphide bonds (Sánchez-Gómez et al., unpublished observations). Therefore, ideally, the modification of cellular proteins by electrophilic eicosanoids should be confirmed by the direct observation of adducts by methods like mass spectrometry.

\section{Proteomic Studies}

Biotinylated analogs of cyPG have been used in several studies to visualize and/or identify protein targets for the modification by cyPG (FIG. 3). We recently observed the binding of biotinylated $15 \mathrm{~d}-\mathrm{PGJ}_{2}$ to approximately 50 target proteins in mesangial cells by $2 \mathrm{D}$ electrophoresis and Western blot with

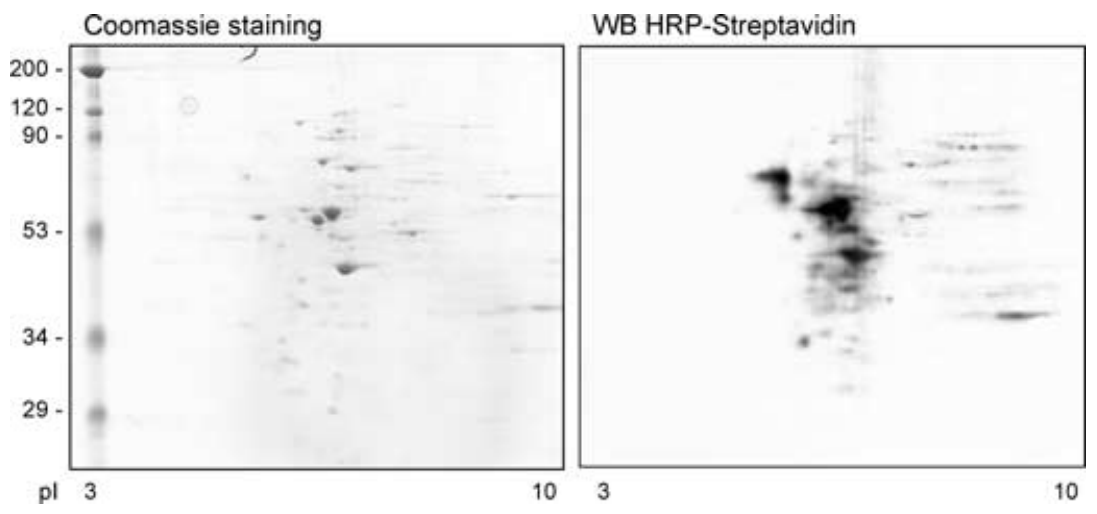

FIGURE 3. Typical pattern of biotinylated $15 \mathrm{~d}-\mathrm{PGJ}_{2}$-modified proteins in rat mesangial cells. Mesangial cells were incubated with $5 \mu \mathrm{M}$ biotinylated $15 \mathrm{~d}-\mathrm{PGJ}_{2}$ for $2 \mathrm{~h}$. Cell lysates were analyzed by 2D electrophoresis and either Coomassie Gold staining or Western blot with horseradish peroxidase streptavidin and ECL. 
horseradish peroxidase-conjugated streptavidin. ${ }^{21}$ Levonen and co-workers, ${ }^{30}$ found nearly 30 proteins modified by biotinylated $15 \mathrm{~d}-\mathrm{PGJ}_{2}$ in human embryonic kidney 293 cells. For these studies to be mechanistically relevant it is important that the biotinylated analogs of electrophilic eicosanoids mimic the biological effects of the nonmodified compounds. In a recent study we have observed that biotinylated $15 \mathrm{~d}-\mathrm{PGJ}_{2}$ displays potent anti-inflammatory effects and induces heat shock in mesangial cells, thus mimicking the effects of $15 \mathrm{~d}-\mathrm{PGJ} \mathrm{J}_{2} \cdot{ }^{59} \mathrm{In}$ a previous study we showed that the ability of $15 \mathrm{~d}-\mathrm{PGJ}_{2}$ to modify cellular proteins was required for the anti-inflammatory effects of this PG. Therefore, we reasoned that the biotinylated compound could be a valid tool to identify novel targets for cyPG, whose modification could play a role in the anti-inflammatory effects of these compounds. We have recently reported the identification of some of the modified proteins, which include proteins involved in the heat shock response and in the organization of the cytoskeleton, such as vimentin and tubulin. ${ }^{59}$ A recent study using also biotinylated $15 \mathrm{~d}$ $\mathrm{PGJ}_{2}$ has identified 17 potential targets of the electrophile responsive proteome in isolated liver mitochondria, thus suggesting that electrophilic lipid oxidation products can target a subproteome in the mitochondria with consequences for cytochrome $\mathrm{c}$ release and induction of apoptosis. ${ }^{64}$ The elucidation of the role of these targets in the biological effects of electrophilic eicosanoids will grant further developments in this field.

\section{Study of the Selectivity of the Modification: Interactions with GSH}

Some cyPG have shown beneficial effects in experimental models of inflammation and tissue injury. Therefore, an important aspect of the study of these compounds is the assessment of their therapeutic potential, which requires a deeper knowledge of their mechanism of action and the identification of novel targets for therapeutic intervention. In this context it is important to ascertain the selectivity of protein modification by cyPG. The studies with biotinylated analogs of cyPG described above have shown that the modification of proteins by these compounds is a selective process, which, in spite of the broad reactivity of cyPG, does not occur randomly but affects a defined subset of cellular proteins. These targets are different from the proteins modified by general cysteine reagents such as biotinylated iodoacetamide. ${ }^{21}$ By using biotinylated $15 \mathrm{~d}-\mathrm{PGJ}_{2}$ and $\mathrm{PGA}_{1}$ we have recently observed that these two cyPG bind to some common targets but also to some selective targets in NIH-3T3 fibroblasts. ${ }^{65}$ Therefore, selectivity of protein modification by cyPG depends in part on the structure of the PG. In addition, the selectivity of the modification can be observed in individual proteins. Both p50 and H-Ras possess several accessible cysteine residues, however, only one cysteine residue is modified by $15 \mathrm{~d}-\mathrm{PGJ}_{2}$ in either protein. ${ }^{24,32}$ In H-Ras, cysteine 184 , out of the six cysteine residues of the protein, has been found to be the main site for $15 \mathrm{~d}-\mathrm{PGJ}_{2}$ 


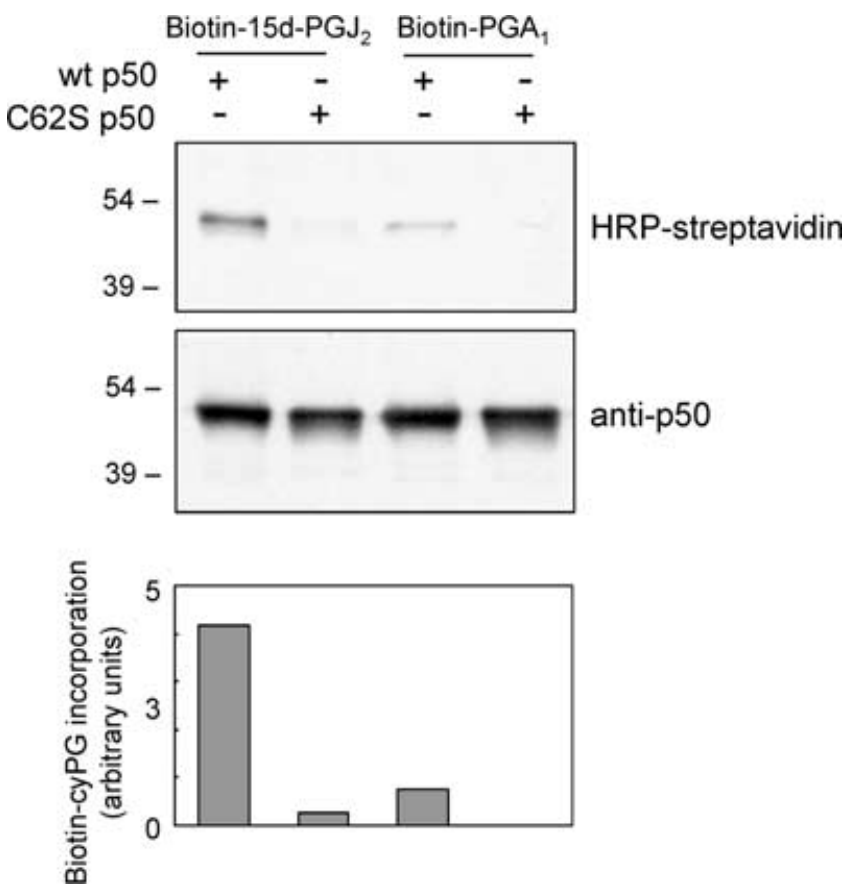

FIGURE 4. Selectivity of the modification of the cysteine residues in p50 by biotinylated $15 \mathrm{~d}-\mathrm{PGJ}_{2}$ and $\mathrm{PGA}_{1}$. Wild type (wt) or cysteine 62 to serine mutant p50 (C62S) at 0.5 $\mu \mathrm{M}$ was incubated with $1 \mu \mathrm{M}$ biotinylated $15 \mathrm{~d}_{-} \mathrm{PGJ}_{2}$ or $\mathrm{PGA}_{1}$ for $1 \mathrm{~h}$ at r.t. Biotin incorporation was monitored by Western blot with horseradish peroxidase-conjugated streptavidin and p50 levels were assessed by Western blot with anti-p50 antibody. The intensity of the biotin signal, normalized by the signal given by the anti-p 50 antibody, expressed in arbitrary units, is shown in the lower panel. Results are representative of four assays.

binding. In addition, the binding of biotinylated analogs of $15 \mathrm{~d}-\mathrm{PGJ}_{2}$ and $\mathrm{PGA}_{1}$ to a p50 DNA binding domain construct containing seven cysteine residues is dependent on the presence of cysteine 62, indicating that this is the main site for cyPG addition (FIG. 4). Identification of the selective targets for modification by cyPG could contribute to the elucidation of the mechanism of action of endogenous eicosanoids, the structure of which is very diverse. Also, this knowledge may aid in the identification of compounds that selectively target certain proteins of therapeutic interest.

In addition to PG structural determinants, several factors could influence the selectivity of protein modification by cyPG. These include factors related to the structure of the protein, either through the establishment of additional interactions with the eicosanoid, ${ }^{42}$ or due to steric factors. In addition, cysteines with low $\mathrm{pKa}$ would be more susceptible to modification, and an acidic $\mathrm{pH}$ in the vicinity of the adduct would contribute to its stability. It is interesting 
to note that interactions of cyPG with proteins can also be influenced by soluble thiols. ${ }^{66}$ It has been reported that the formation of GSH-CyPG adducts is a reversible process while cyPG addition to proteins appears to be virtually irreversible under physiological conditions. It is well known that GSH is an important factor in the biological effects of cyPG. Depletion of GSH has been associated in several studies with a potentiation of the effects of cyPG. ${ }^{67,68} \mathrm{In}$ contrast, supplementation of cells with N-acetyl-cysteine, as a thiol-protective agent, reduces the effects of cyPG. ${ }^{69}$ Therefore, there seems to exist an inverse correlation between intracellular levels of GSH and the intensity of the biological effects of cyPG. This can be due to several mechanisms, including the ability of GSH to form adducts with cyPG. This can occur both enzymatically, through the action of glutathione- $S$-transferase (GST) enzymes, as well as nonenzymatically. GSH-cyPG adducts are substrates for the multidrug transporters, which play an important role in the detoxification of cyPG. We have observed recently that agents that modulate intracellular GSH levels differentially affect the intensity of the binding of biotinylated cyPG, $15 \mathrm{~d}-\mathrm{PGJ}_{2}$ and $\mathrm{PGA}_{1}$ to cellular proteins. ${ }^{65}$ Biotinylated $15 \mathrm{~d}_{-} \mathrm{PGJ}_{2}$ was more effective than $\mathrm{PGA}_{1}$ at binding cellular proteins. Depletion of GSH with buthionine sulfoximine increased protein modification. Conversely, supplementation with $\mathrm{N}$-acetyl-cysteine reduced the extent of cyPG addition. These changes were more marked in the case of biotinylated $\mathrm{PGA}_{1}$. In addition, the modulation of the binding of cyPG by GSH was not uniform for all polypeptides, thus leading to alterations in the pattern of labeled proteins. These results suggest that GSH levels may contribute not only to the intensity but also to the selectivity of protein modification by biotinylated cyPG. These effects may be related to the differential reactivity of two cyPG toward soluble thiols. The $15 \mathrm{~d}-\mathrm{PGJ}_{2}$ presents a dienone structure, while $\mathrm{PGA}_{1}$ is a single enone. Adducts of single enone cyPG and GSH have been reported to be more stable than dienone cyPG-GSH adducts. ${ }^{66}$ This may be the reason why GSH levels affect the binding of biotinylated-PGA 1 to proteins more intensely. However, as outlined above, the effect of GSH does not affect uniformly all proteins. We have addressed this issue by exploring the effect of GSH on the incorporation of biotinylated cyPG to several purified proteins in vitro. We have observed that GSH reduces the modification of GST, but it can improve the modification of p50 (Gayarre et al., unpublished observations). These opposite effects could be due to several mechanisms. GSH or GSH conjugates can alter the conformational status of GST modifying the orientation of critical cysteine residues. ${ }^{70}$ This could preclude binding of cyPG. On the other hand, our results indicate that GSH may favor the incorporation of cyPG into certain proteins by contributing to the maintenance of critical cysteine residues in a reduced state, and therefore, accessible for modification. Extensive protein oxidation due to oxidative stress or GSH depletion would then lead to an inhibition of the incorporation of cyPG and probably to an attenuation of cyPG-specific effects. It is interesting to consider that under situations of oxidative stress or increased 
NO generation, the GSSG or GSNO formed can act as thiolating agents and induce reversible protein glutathiolation. In several proteins, for instance, in c-Jun and p50, the cysteine residues that are targets for modification by cyPG are also targets for glutathiolation. Therefore, these two types of agents, oxidant species derived from GSH and cyPG, may compete for binding to the same residue(s). In fact, glutathiolation under these conditions, could act as a defense mechanism protecting critical cysteine residues in signaling proteins from the irreversible addition of cyPG, as it has been proposed previously in the context of oxidative or nitrosative stress. ${ }^{71}$

The effects of GSH levels on the biological actions of electrophilic eicosanoids could be mediated by an additional mechanism. It has been recently reported that certain synthetic cysteine adducts of cyclopentenones can display potent biological activity. ${ }^{72}$ In addition, it is well known that GSH adducts of several endogenous oxyeicosanoids retain biological activity or can even display novel actions. ${ }^{73}$ Therefore, the formation of cyPG-GSH adducts could give rise to the appearance of biochemical species with effects and mechanisms of action different from those of the parent cyPG.

\section{Subcellular Localization of Cellular Targets for Electrophilic Eicosanoids}

The early studies with radioactive cyPG already detected important differences in the subcellular distribution of cyPG depending on their structure, with single enone cyPG being present as soluble species in a higher proportion than dienone cyPG. ${ }^{56}$ The subcellular fate of cyPG has also been explored by means of biotinylated or fluorescent analogs of cyPG. These studies have shown cell type-dependent differences in the subcellular distribution of cyPG. In HeLa cells incubated with biotinylated $15 \mathrm{~d}-\mathrm{PGJ}_{2}$, a predominantly nuclear staining has been observed ${ }^{74}$ while in mesangial cells both nuclear and cytosolic compartments show biotin staining. ${ }^{21}$ These observations may correlate with some of the biological effects of cyPG described in both cell types. $15 \mathrm{~d}-\mathrm{PGJ}_{2}$ inhibits NF- $\mathrm{BB}$ activation in both systems. However, the mechanism of inhibition appears to involve mainly nuclear events in HeLa cells, ${ }^{24,26}$ and both cytoplasmic and nuclear processes in macrophages ${ }^{26}$ and in mesangial cells. ${ }^{75}$ Interestingly, GSH levels and/or GST activity have been proposed to play an important role in the predominance of either cytosolic or nuclear effects of cyPG. ${ }^{26,53}$ It is tempting to speculate that these factors could also influence the cellular compartment in which cyPG would preferentially bind to their targets. A recent study using a fluorescent derivative of $15 \mathrm{~d}-\mathrm{PGJ}_{2}$ indicates that this compound localizes significantly to the mitochondria in endothelial cells. ${ }^{58}$ It has been proposed that interaction with mitochondrial proteins contributes to the oxidative stress induced by this compound. ${ }^{58}$ 


\section{Induction of Protein Cross-Links}

One of the mechanisms by which electrophilic lipids can damage proteins is by inducing protein cross-links. This appears particularly relevant in the case of electrophilic aldehydes, such as 4-hydroxy-nonenal, isoketals, and levuglandins. ${ }^{16}$ The consequence of this modification can be the accumulation of the modified proteins that cannot be degraded by the proteasome, and the formation of anomalous aggregates that may damage cells and contribute to the pathophysiology of several processes, including inflammatory diseases and neurodegeneration. ${ }^{16}$ It is interesting to note that certain cyPG possess a dienone structure, and therefore they could form Michael adducts with two cysteine residues. In fact, 9-deoxy- $\Delta^{9}, \Delta^{12}(\mathrm{E})-\mathrm{PGD}_{2}$, which possesses two electrophilic carbons, has been described to form a conjugate with two molecules of GSH. ${ }^{76}$ In this context, we have recently described that $15 \mathrm{~d}-\mathrm{PGJ}_{2}$, a dienone, can induce cross-linking of the protein c-Jun, ${ }^{27}$ thus providing the first example of protein cross-linking induced by cyPG. This effect could also be observed in mutant c-Jun constructs which only possess one cysteine residue, like the C320S c-Jun mutant, which only presents the cysteine residue located in the DNA binding domain, cysteine 269 (FIG. 5). The dimer formed by treatment with $15 \mathrm{~d}-\mathrm{PGJ}_{2}$ was resistant to electrophoresis under denaturing and reducing conditions. In contrast, treatment with $\mathrm{H}_{2} \mathrm{O}_{2}$ induced the dimerization of C320S c-Jun, which was reversed by incubation with dithiothreitol (DTT). In addition, in assays performed with biotinylated $15 \mathrm{~d}-\mathrm{PGJ}_{2}$ we could observe the presence of biotin signal in the c-Jun dimer. Taken together, these observations are strongly indicative of the participation of $15 \mathrm{~d}_{-} \mathrm{PGJ}_{2}$ as the cross-linking bridge in the oligomer. Therefore, it would be important to assess whether other electrophilic lipids with dienone structure can induce a similar phenomenon in c-Jun or other proteins and what could be its functional relevance.

\section{CONCLUSIONS AND PERSPECTIVES}

Electrophilic eicosanoids are gaining importance both as signaling molecules and as potential pharmacological agents. In recent years, the work of numerous research groups has greatly broadened our knowledge of their generation, mechanisms of action, and pathophysiological and/or biochemical importance. The use of prostanoids with cyclopentenone structure in diverse experimental approaches has allowed exploring the subcellular localization, target identity, structural implications and functional consequences of the modification of cellular proteins by electrophilic eicosanoids (FIG. 6). In spite of this there are many aspects of the biology of these lipids that remain to be elucidated and will probably be the subject of intense research in the future. Much needs to be learned about the potential selectivity of protein modification by eicosanoids and its importance in their biological actions. It would be also 


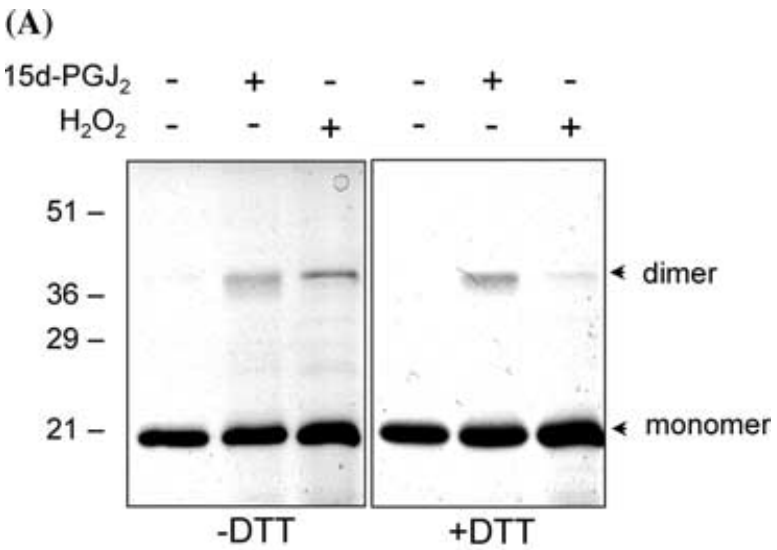

(B)

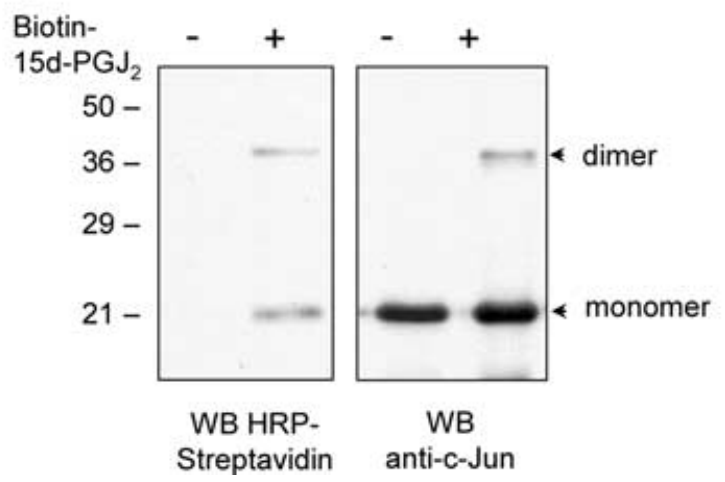

FIGURE 5. Involvement of $15 \mathrm{~d}_{-}-\mathrm{PGJ}_{2}$ in c-Jun cross-linking. (A) A C320S mutant c-Jun construct was incubated the presence of $15 \mathrm{~d}-\mathrm{PGJ}_{2}(10 \mu \mathrm{M})$ or $\mathrm{H}_{2} \mathrm{O}_{2}(1 \mathrm{mM})$ for $30 \mathrm{~min}$ at $37^{\circ} \mathrm{C}$. Incubation mixtures were treated with $50 \mathrm{mM}$ iodoacetamide before of after treatment with $10 \mathrm{mM}$ DTT, as indicated, and analyzed by nonreducing SDS-PAGE and Coomassie staining. The position of c-Jun monomer and dimer is indicated. (B) cJun C320S was incubated in the absence or presence of biotinylated $15 \mathrm{~d}-\mathrm{PGJ}_{2}$, subjected to SDS-PAGE under reducing conditions and analyzed by Western blot with horseradish peroxidase-conjugated streptavidin to reveal biotinylated $15 \mathrm{~d}-\mathrm{PGJ}_{2}$ incorporation, and with anti-c-Jun antibody to show the position of c-Jun monomer and dimer.

interesting to explore the potential reversibility of these modifications in some proteins, or their effects on protein turnover. The elucidation of the physiological or pathophysiological roles of some electrophilic eicosanoids, such as cyPG, will require a detailed study of their generation in diverse situations and the use of sensitive methods for isolation and quantitation. In this context, a significant challenge will be the detection and identification of proteins modified by endogenous eicosanoids. In parallel, it would be important 


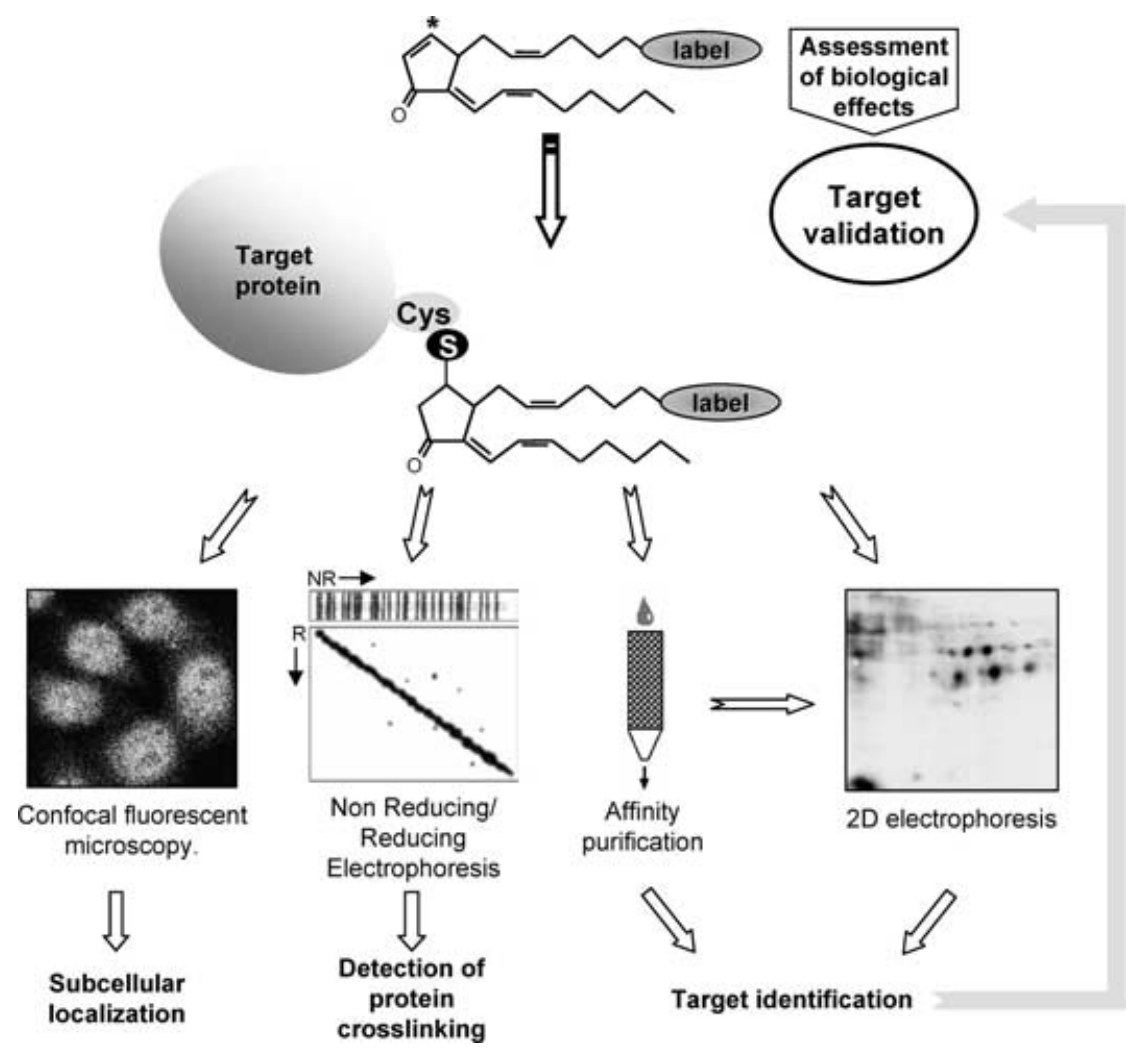

FIGURE 6. Diverse experimental approaches employed in the characterization of the electrophilic eicosanoid-protein interactome through the use of cyPG derivatives.

to continue the assessment of the therapeutic potential of cyPG-based active species.

\section{ACKNOWLEDGMENTS}

We wish to acknowledge the work of collaborators who have contributed to the research on this topic, F. J. Sánchez-Gómez, J. Gayarre, D. Sánchez, M. I. Avellano, Drs. E. Cernuda-Morollón, E. Pineda-Molina, F. J. Cañada, L. Boscá, and J.M. Rojas. The technical assistance of M. J. Carrasco is gratefully acknowledged. Our work is supported by Grants SAF2003-03713 from Ministerio de Educación y Ciencia and 0179 from Fundación La Caixa. K.S. is the recipient of an I3P fellowship from CSIS. 


\section{REFERENCES}

1. MARnett, L.J., J.N. Riggins \& J.D. WeSt. 2003. Endogenous generation of reactive oxidants and electrophiles and their reactions with DNA and proteins. J. Clin. Invest. 111: 583-593.

2. Fratelli, M. et al. 2002. Identification by redox proteomics of glutathionylated proteins in oxidatively stressed human T lymphocytes. Proc. Natl. Acad. Sci. USA 99: $3505-3510$.

3. JAFFREY, S.R. et al. 2001. Protein S-nitrosylation: a physiological signal for neuronal nitric oxide. Nat. Cell Biol. 3: 193-197.

4. Scozzafava, A., A. CASini \& C.T. Supuran. 2002. Targeting cysteine residues of biomolecules: new approaches for the design of antiviral and anticancer drugs. Curr. Med. Chem. 9: 1167-1185.

5. FUnK, C.D. 2001. Prostaglandins and leukotrienes: advances in eicosanoid biology. Science 294: 1871-1875.

6. KuHN, H. 2005. Biologic relevance of lipoxygenase isoforms in atherogenesis. Expert Rev. Cardiovasc. Ther. 3: 1099-1110.

7. Roberts, L.J., 2nd, J.P. Fessel \& S.S. DAvies. 2005. The biochemistry of the isoprostane, neuroprostane, and isofuran pathways of lipid peroxidation. Brain Pathol. 15: 143-148.

8. GAO, L. et al. 2003. Formation of prostaglandins $\mathrm{E}_{2}$ and $\mathrm{D}_{2}$ via the isoprostane pathway: a mechanism for the generation of bioactive prostaglandins independent of cyclooxygenase. J. Biol. Chem. 278: 28479-28489.

9. Straus, D.S. \& C.K. Glass. 2001. Cyclopentenone prostaglandins: new insights on biological activities and cellular targets. Med. Res. Rev. 21: 185210.

10. PÉRez-Sala, D. \& S. LAmas. 2001. Regulation of cyclooxygenase-2 expression by nitric oxide in cells. Antioxid. Redox Signal. 3: 231-248.

11. PÉREZ-SALA, D. et al. 2001. Posttranscriptional regulation of human iNOS by the NO/cGMP pathway. Am. J. Physiol. Renal Physiol. 280: F466-F473.

12. Serhan, C.N. \& J. SAVILl. 2005. Resolution of inflammation: the beginning programs the end. Nat. Immunol. 6: 1191-1197.

13. Díaz-Cazorla, M., D. PÉrez-Sala \& S. Lamas. 1999. Dual effect of nitric oxide donors on cyclooxygenase- 2 expression in human mesangial cells. J. Am. Soc. Nephrol. 10: 943-952.

14. LEVY, B.D. 2001. Lipid mediator class switching during acute inflammation: signals in resolution. Nat. Immunol. 2: 612-619.

15. Roberts, L.J., 2nd \& J.D. Morrow. 2002. Products of the isoprostane pathway: unique bioactive compounds and markers of lipid peroxidation. Cell. Mol. Life Sci. 59: 808-820.

16. DAVIES, S.S. et al. 2002. Effects of reactive gamma-ketoaldehydes formed by the isoprostane pathway (isoketals) and cyclooxygenase pathway (levuglandins) on proteasome function. FASEB J. 16: 715-717.

17. GILROY, D.W. et al. 2004. Inflammatory resolution: new opportunities for drug discovery. Nat. Rev. Drug Discov. 3: 401-416.

18. GiLroy, D.W. et al. 1999. Inducible cyclooxygenase may have anti-inflammatory properties. Nat. Med. 5: 698-701.

19. FormAn, B.M. et al. 1995. 15-Deoxy- $\Delta^{12,14}$-prostaglandin $J_{2}$ is a ligand for the adipocyte determination factor PPAR gamma. Cell 83: 803-812. 
20. ReILly, C.M. et al. 2001. Prostaglandin $\mathrm{J}_{2}$ inhibition of mesangial cell iNOS expression. Clin. Immunol. 98: 337-345.

21. SÁNCHEZ-GómEZ, F.J. et al. 2004. Protein thiol modification by 15 -deoxy- $\Delta^{12,14}$ prostaglandin $\mathrm{J}_{2}$ addition in mesangial cells: role in the inhibition of proinflammatory genes. Mol. Pharmacol. 66: 1349-1358.

22. CAstrillo, A. et al. 2000. Inhibition of IkappaB kinase and IkappaB phosphorylation by 15 -deoxy- $\Delta^{12,14}$-prostaglandin $\mathbf{J}_{2}$ in activated murine macrophages. Mol. Cell. Biol. 20: 1692-1698.

23. Rossi, A. et al. 2000. Anti-inflammatory cyclopentenone prostaglandins are direct inhibitors of IкB kinase. Nature 403: 103-108.

24. CernudA-Morollón, E. et al. 2001. 15-Deoxy- $\Delta^{12,14}$-prostaglandin $\mathrm{J}_{2}$ inhibition of NF- $\kappa$ B DNA binding through covalent modification of the p50 subunit. J. Biol. Chem. 276: 35530-35536.

25. ShibAtA, T. et al. 2003. An endogenous electrophile that modulates the regulatory mechanism of protein turnover: inhibitory effects of 15 -deoxy- $\Delta 12,14$ prostaglandin $\mathrm{J}_{2}$ on proteasome. Biochemistry 42: 13960-13968.

26. Straus, D.S. et al. 2000. 15-deoxy- $\Delta^{12,14}$-prostaglandin $\mathrm{J}_{2}$ inhibits multiple steps in the NF-кB signaling pathway. Proc. Natl. Acad. Sci. USA 97: 48444849.

27. Pérez-Sala, D., E. Cernuda-Morollón \& F.J. Cañada. 2003. Molecular basis for the inhibition of AP-1 DNA binding by 15 -deoxy- $\Delta^{12,14}$-prostaglandin $\mathrm{J}_{2}$. J. Biol. Chem. 278: 51251-51260.

28. ShibAtA, T. et al. 2003. Thioredoxin as a molecular target of cyclopentenone prostaglandins. J. Biol. Chem. 278: 26046-26054.

29. Moos, P.J. et al. 2003. Electrophilic prostaglandins and lipid aldehydes repress redox-sensitive transcription factors p53 and hypoxia-inducible factor by impairing the selenoprotein thioredoxin reductase. J. Biol. Chem. 278: 745-750.

30. LeVONEN, A.L. et al. 2004. Cellular mechanisms of redox cell signaling: the role of cysteine modification in controlling antioxidant defenses in response to electrophilic lipid oxidation products. Biochem. J. 378: 373-382.

31. Ітон, K. et al. 2004. Transcription factor Nrf2 regulates inflammation by mediating the effect of 15-deoxy- $\Delta^{12,14}$-prostaglandin $\mathrm{J}_{2}$. Mol. Cell. Biol. 24: 36-45.

32. Oliva, J.L. et al. 2003. The cyclopentenone 15 -deoxy- $\Delta^{12,14}$-prostaglandin $\mathrm{J}_{2}$ binds to and activates H-Ras. Proc. Natl. Acad. Sci. USA 100: 4772-4777.

33. Wagner, T.M., J.E. Mullally \& F.A. FitzPatrick. 2006. Reactive lipid species from cyclooxygenase-2 inactivate tumor suppressor LKB1 / STK11: cyclopentenone prostaglandins and 4-hydroxy-2-nonenal covalently modify and inhibit the AMP-kinase kinase that modulates cellular energy homeostasis and protein translation. J. Biol. Chem. 281: 2598-2604.

34. ChAtTERJEe, P.K. et al. 2004. The cyclopentenone prostaglandin 15 -deoxy- $\Delta^{12,14}$ prostaglandin $\mathrm{J}_{2}$ ameliorates ischemic acute renal failure. Cardiovasc. Res. 61: 630-643.

35. KaWAHITO, Y. et al. 2000. 15-deoxy- $\Delta^{12,14}-\mathrm{PGJ}_{2}$ induces synoviocyte apoptosis and suppresses adjuvant-induced arthritis in rats. J. Clin. Invest. 106: 189-197.

36. DiAB, A. et al. 2002. Peroxisome proliferator-activated receptor-gamma agonist 15-deoxy- $\Delta^{12,14}$-prostaglandin $\mathrm{J}_{2}$ ameliorates experimental autoimmune encephalomyelitis. J. Immunol. 168: 2508-2515.

37. IANARO, A. et al. 2003. 2-Cyclopenten-1-one and prostaglandin $\mathrm{J}_{2}$ reduce restenosis after balloon angioplasty in rats: role of NF-кB. FEBS Lett. 553: 21-27. 
38. ANDO, M. et al. 2003. Retrovirally introduced prostaglandin $\mathrm{D}_{2}$ synthase suppresses lung injury induced by bleomycin. Am. J. Respir. Cell. Mol. Biol. 28: 582-591.

39. MusieK, E.S. et al. 2005. Cyclopentenone eicosanoids as mediators of neurodegeneration: a pathogenic mechanism of oxidative stress-mediated and cyclooxygenase-mediated neurotoxicity. Brain Pathol. 15: 149-158.

40. Chen, Y., J.D. Morrow \& L.J. RoberTs, 2nd. 1999. Formation of reactive cyclopentenone compounds in vivo as products of the isoprostane pathway. J. Biol. Chem. 274: 10863-10868.

41. MusieK, E. et al. 2005. Cyclopentenone isoprostanes inhibit the inflammatory response in macrophages. J. Biol. Chem. 280: 35562-35570.

42. SHIRAKI, T. et al. 2005. a,ß-Unsaturated ketone is a core moiety of natural ligands for covalent binding to peroxisome proliferator-activated receptor. J. Biol. Chem. 280: $14145-14153$.

43. SubBanagounder, G. et al. 2002. Epoxyisoprostane and epoxycyclopentenone phospholipids regulate monocyte chemotactic protein- 1 and interleukin- 8 synthesis. Formation of these oxidized phospholipids in response to interleukin1beta. J. Biol. Chem. 277: 7271-7281.

44. BiRUKOV, K.G. et al. 2004. Epoxycyclopentenone-containing oxidized phospholipids restore endothelial barrier function via Cdc42 and Rac. Circ. Res. 95: 892-901.

45. DianZAni, M.U. 2003. 4-hydroxynonenal from pathology to physiology. Mol. Aspects Med. 24: 263-272.

46. KeHrer, J.P. \& S.S. Biswal. 2000. The molecular effects of acrolein. Toxicol. Sci. 57: $6-15$.

47. CEASER, E.K. et al. 2004. Mechanisms of signal transduction mediated by oxidized lipids: the role of the electrophile-responsive proteome. Biochem. Soc. Trans. 32: $151-155$.

48. KwoK, B.H. et al. 2001. The anti-inflammatory natural product parthenolide from the medicinal herb Feverfew directly binds to and inhibits ІкB kinase. Chem. Biol. 8: 759-766.

49. LEE, J.H. et al. 2002. Kaurane diterpene, kamebakaurin, inhibits NF-кB by directly targeting the DNA-binding activity of p50 and blocks the expression of antiapoptotic NF-кB target genes. J. Biol. Chem. 277: 18411-18420.

50. Mahon, T.M. \& L.A. O’NeILl. 1995. Studies into the effect of the tyrosine kinase inhibitor herbimycin A on NF- $\mathrm{B}$ activation in T lymphocytes. Evidence for covalent modification of the p50 subunit. J. Biol. Chem. 270: 2855728564.

51. OgINo, S. et al. 2004. Herbimycin A abrogates Nuclear Factor-kappaB activation by interacting preferentially with the IkappaB Kinase beta subunit. Mol. Pharmacol. 65: $1344-1351$.

52. VERBITSKI, S.M. et al. 2004. Punaglandins, chlorinated prostaglandins, function as potent Michael receptors to inhibit ubiquitin isopeptidase activity. J. Med. Chem. 47: 2062-2070.

53. NOYORI, R. \& M. SUZUKI. 1993. Organic synthesis of prostaglandins: advancing biology. Science 259: 44-45.

54. NARUMIYA, S. \& M. FuKUSHIMA. 1986. Site and mechanism of growth inhibition by prostaglandins. I. Active transport and intracellular accumulation of cyclopentenone prostaglandins, a reaction leading to growth inhibition. J. Pharmacol. Exp. Ther. 239: 500-505. 
55. NARUMiYA, S. et al. 1986. Site and mechanism of growth inhibition by prostaglandins. II. Temperature-dependent transfer of a cyclopentenone prostaglandin to nuclei. J. Pharmacol. Exp. Ther. 239: 506-511.

56. NARUmiYA, S. et al. 1987. Site and mechanism of growth inhibition by prostaglandins. III. Distribution and binding of prostaglandin $A_{2}$ and $\Delta^{12}$ prostaglandin $\mathrm{J}_{2}$ in nuclei. J. Pharmacol. Exp. Ther. 242: 306-311.

57. PARKer, J. 1995. Prostaglandin $A_{2}$ protein interactions and inhibition of cellular proliferation. Prostaglandins 50: 359-375.

58. LANDAR, A. et al. 2006. The interaction of electrophilic lipid oxidation products with mitochondria in endothelial cells and the formation of reactive oxygen species. Am. J. Physiol. Heart Circ. Physiol. 290: H1777-H1787.

59. Stamatakis, K., F.J. SÁnchez-Gómez \& D. PÉrez-Sala. 2006. Identification of novel protein targets for modification by 15 -deoxy- $\Delta^{12,14}$-prostaglandin $\mathrm{J}_{2}$ in mesangial cells reveals multiple interactions with the cytoskeleton. J. Am. Soc. Nephrol. 17: 89-98.

60. Hortelano, S. et al. 2000. Contribution of cyclopentenone prostaglandins to the resolution of inflammation through the potentiation of apoptosis in activated macrophages. J. Immunol. 165: 6525-6531.

61. Kondo, M. et al. 2001. Cyclopentenone prostaglandins as potential inducers of intracellular oxidative stress. J. Biol. Chem. 276: 12076-12083.

62. LI, L. et al. 2001. 15-deoxy- $\Delta^{12,14}$-prostaglandin $\mathrm{J}_{2}$ induces apoptosis of human hepatic myofibroblasts. A pathway involving oxidative stress independently of peroxisome-proliferator-activated receptors. J. Biol. Chem. 276: 38152-38158.

63. IsHII, T. \& K. UCHIDA. 2004. Induction of reversible cysteine-targeted protein oxidation by an endogenous electrophile 15 -deoxy- $\Delta^{12,14}$-prostaglandin $\mathrm{J}_{2}$. Chem. Res. Toxicol. 17: 1313-1322.

64. LANDAR, A. et al. 2006. Induction of the permeability transition and cytochrome c release by 15-deoxy prostaglandin $\mathrm{J}_{2}$ in mitochondria. Biochem. J. 394: 185-195.

65. GAYARRE, J. et al. 2005. Differential selectivity of protein modification by the cyclopentenone prostaglandins $\mathrm{PGA}_{1}$ and 15 -deoxy- $\Delta^{12,14}-\mathrm{PGJ}_{2 \text { : }}$ role of glutathione. FEBS Lett. 579: 5803-5808.

66. SuzUKI, M. et al. 1997. Chemical implications for antitumor and antiviral prostaglandins: reaction of $\Delta^{7}$-prostaglandin $A_{1}$ and prostaglandin $A_{1}$ methyl esters with thiols. J. Am. Chem. Soc. 119: 2376-2385.

67. Atsmon, J. et al. 1990. Conjugation of 9-deoxy- $\Delta^{9}, \Delta^{12}(\mathrm{E})$-prostaglandin $\mathrm{D}_{2}$ with intracellular glutathione and enhancement of its antiproliferative activity by glutathione depletion. Cancer Res. 50: 1879-1885.

68. LeVonen, A.L. et al. 2001. Biphasic effects of 15-deoxy- $\Delta^{12,14}$-prostaglandin $\mathrm{J}_{2}$ on glutathione induction and apoptosis in human endothelial cells. Arterioscler. Thromb. Vasc. Biol. 21: 1846-1851.

69. ZhANG, X. et al. 2004. Stress protein activation by the cyclopentenone prostaglandin 15-deoxy- $\Delta^{12,14}$-prostaglandin $\mathrm{J}_{2}$ in human mesangial cells. Kidney Int. 65: 798-810.

70. VEGA, M.C. et al. 1998. The three-dimensional structure of Cys-47-modified mouse liver glutathione S-transferase P1-1. Carboxymethylation dramatically decreases the affinity for glutathione and is associated with a loss of electron density in the alphaB-310B region. J. Biol. Chem. 273: 2844-2850.

71. KLATt, P. \& S. LAMAS. 2000. Regulation of protein function by S-glutathiolation in response to oxidative and nitrosative stress. Eur. J. Biochem. 267: 49284944. 
72. BiCKLEY, J.F. et al. 2004. Reactions of some cyclopentenones with selected cysteine derivatives and biological activities of the product thioethers. Bioorg. Med. Chem. 12: 3221-3227.

73. Murphy, R.C. \& S. ZARINI. 2002. Glutathione adducts of oxyeicosanoids. Prostaglandins Other Lipid Mediat. 68-69: 471-482.

74. Stamatakis, K., F.J. SÁnChez-Gómez \& D. PÉRez-SAla. 2004. Protein modification by cyclopentenone prostaglandin addition: biological actions and therapeutic implications. Gene Ther. Mol. Biol. 8: 241-258.

75. Cernuda-Morollón, E. \& D. PÉrez-Sala. 2006. Regulation of proinflammatory transcription factors by direct modification with cyclopentenone prostaglandins. In Trends in DNA Research. C.R. Woods, Ed. 1-31. Nova Science Publishers, Inc. Hauppauge, NY.

76. Atsmon, J. et al. 1990. Formation of thiol conjugates of 9-deoxy- $\Delta^{9}, \Delta^{12}(E)$ prostaglandin $\mathrm{D}_{2}$ and $\Delta^{12}(E)$-prostaglandin $\mathrm{D}_{2}$. Biochemistry 29: 3760-3765. 